\title{
Antwort auf den Leserbrief von T. Bertsch und T. Hirsch zu: Zetzmann K, Ludolph I, Horch R, et al. Bildgebende Diagnostik zur Therapieplanung bei Lip- und Lymphödem. Phlebologie 2020, 2(49): 72-78
}

Gegenstand der Diskussion ist der Nachdruck (Phlebologie 2020, 2(49): 72-78) des Thieme Verlags einer Originalartikels aus HaMiPla für die Plastischen Chirurgen 2018 erschienen (Zetzmann K, Ludolph I, Horch RE, Boos AM. Handchir Mikrochir Plast Chir. 2018 Dec; 50(6): 386-392. doi:10.1055/ a-0739-7911. Epub 2019 Jan 8. PMID: 30620 976).

Die Arbeit fokussiert sich dabei auf mögliche bildgebende Diagnostik zur Therapieplanung bei Lipödem- und LymphödemPatienten. Die Autoren des Leserbriefs merken an, dass Lipödem und Lymphödem „beides gänzlich unterschiedliche Erkrankungen - mit unterschiedlicher Pathogenese, unterschiedlicher Klinik, unterschiedlichen Komplikationsoptionen und vor allem unterschiedlicher Therapie " seien und der in dem hier diskutierten Artikel mehrfach verwendete Begriff Lipo-Lymphödem irreführend sei. Auch wenn wir in unserer Arbeit entgegen dem Vortrag der Kollegen keineswegs die Meinung vertreten, dass es sich beim Lipödem und Lymphödem um dieselbe Erkrankung, wie von den Leserbriefautoren angenommen, handelt, erlauben wir uns dennoch den Hinweis, dass der Begriff Lipo-Lymphödem in der wissenschaftlichen Literatur ungeachtet der Leserzuschrift weiterhin existiert [2-4]. Trotz einer bekanntermaßen bestehenden Unschärfe hinsichtlich des Terminus „LipoLymphödem“ ist dieser Begriff konform mit der 2018 interdisziplinär erstellten und nach wie vor gültigen Leitlinie (AWMF Registernummer 037-012).

Unsere Arbeit zur bildgebenden Diagnostik diskutiert nicht die Pathogenese beider Erkrankungen und auch nicht mögliche Überschneidungen. Die reine Verwendung des Begriffs „Lipödem“ kann daher nicht als Fehlverständnis der Autoren gedeutet werden, da weder eine (Neu)Definition des Ödems oder deren Stadieneinteilung, sondern vielmehr Grundlagen der bildgebenden Diagnostik gemäß aktuellen wissenschaftlichen Erkenntnissen dargelegt werden. Das adipositasassoziierte Lymphödem ist auch in unserem bariatrischen Patientengut anzutreffen und
Gewichtskontrolle und psychosoziale Begleitung stellen zentrale Elemente im Rahmen der Behandlung in einem AdipositasZentrum dar. Der enge interdisziplinäre Austausch zwischen Viszeralchirurgen, Ernährungstherapeuten, Psychotherapeuten, Sozialarbeitern und Selbsthilfegruppen ist gerade in universitären Zentren gelebte Praxis.

Bei insgesamt noch schwacher Evidenz, die Pathophysiologie und Therapie des Lipödems betreffend, erscheint uns der Leserbrief wie auch die Arbeiten zu den
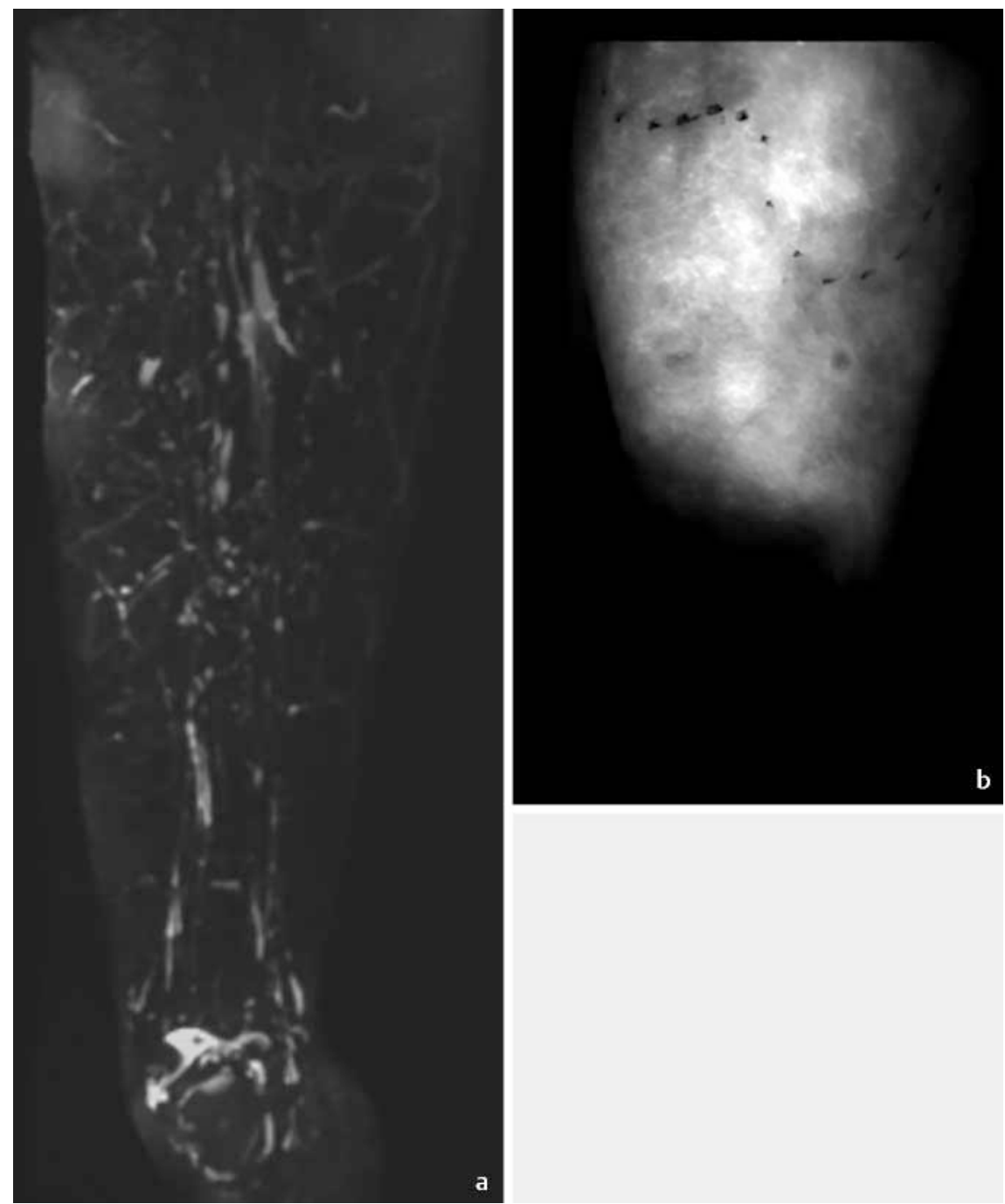

- Abb. 1 A Lipödem Unterschenkel im MRT mit Zeichen vermehrter Flüssigkeitsansammlung im Gewebe. B ICG-Lymphografie mit Injektion im Zwischenzehenraum „Stardust“ und diffuses Verteilungsmuster des ICG am Unterschenkel. 
Erkenntnissen Expertenmeinungen zu fundieren. Daher scheint uns der Begriff „Paradigmenwechsel“ beim Lipödem verfrüht und wir können die Autoren nur animieren, den evidenzbasierten Weg zu fördern.

Wir unterstützen die Behandlung des Weichteilschmerzes, der psychischen Vulnerabilität und die begleitende Behandlung der Adipositas. Wir sind gespannt auf die angekündigte Veröffentlichung des Konsensus-Papiers „European Best Practice of Lipoedema“.

Die Aussage „Weder klinische noch bildgebende Untersuchungen konnten jemals relevante Flüssigkeitsmengen im Gewebe bei Lipödem-Patientinnen nachweisen" verwundert etwas und sollte besser im wissenschaftlichen Kontext betrachtet werden. Wir führen beispielsweise bildgebende Diagnostik zur Planung von chirurgischen Eingriffen auch bei Lipödem-Patienten durch und konnten durchaus wie auch andere Autoren vermehrte Flüssigkeitsansammlung im MRT und in der ICG-Lymphografie nachweisen ( $\triangleright$ Abb.1). Um die Wertigkeit dieser Methoden weiter zu prüfen, sind unseres Erachtens Untersuchungen an einer randomisierten Patientengruppe sinnvoll und empfehlenswert, um unseren Patienten künftig mit noch mehr Wissen um die Pathophysiologie helfen zu können

Wie nun eine immer wieder klinischerseits zu beobachtende Schwellung/Ödemneigung oder ähnlich beim Lipödem abschließend zu bewerten ist, kann nach dem Kenntnisstand der aktuellen Literatur unseres Erachtens derzeit noch nicht zufriedenstellend beantwortet werden. Auch ist zu beachten, dass die Patientenklientel, die sich bei konservativen und operativ ausgerichteten Ambulanzen vorstellt, durchaus different in der Präsentation der klinischen Beschwerden sein kann, was unter anderem die rege Diskus- sion zu den angeführten „Mythen des Lipödems" deutlich macht.

Zusammenfassend ist insbesondere ein kollegialer und interdisziplinärer Konsens auf Augenhöhe unter Einbeziehung konservativ und operativ ausgerichteter Therapeuten erforderlich. Neben der Berücksichtigung sämtlicher Komorbiditäten und unter Hinzuziehung von Aspekten der Psychologie sollte an einer fortwährenden, wertfreien Aufarbeitung des Krankheitsbildes Lipödem gearbeitet werden, um das Wohl und die bestmögliche Therapie für unsere Patienten gemäß dem aktuellen Wissensstand zu erreichen.

\section{Interessenkonflikt}

Die Autorinnen/Autoren geben an, dass kein Interessenkonflikt besteht.

Autorinnen/Autoren

Anja M. Boos ${ }^{1,2}$, Ingo Ludolph², Raymund E. Horch ${ }^{2}$

${ }^{1}$ Klinik für Plastische Chirurgie, Hand- und Verbrennungschirurgie, Universitätsklinikum Aachen, RWTH Aachen, Aachen

2 Plastisch- und Handchirurgische Klinik, Universitätsklinikum Erlangen, FriedrichAlexander Universität Erlangen-Nürnberg, Erlangen

\section{Korrespondenzadresse}

\section{PD Dr. med. Anja M. Boos}

Klinik für Plastische Chirurgie, Hand- und Verbrennungschirurgie, Universitätsklinikum Aachen, RWTH Aachen

Pauwelsstraße 30

52074 Aachen

aboos@ukaachen.de
Literatur

[1] S1-Leitlinie Lipödem AWMF Registernummer 037-012.

[2] Thielitz A, Bellutti M, Bonnekoh B et al. Progressive lipo-lymphedema associated with increased activity of dermal fibroblasts in monoclonal gammopathy of undetermined significance: is there a causal relationship? Lymphology 2012; 45 (3): 124-129. PMID:23342932

[3] Wollina U, Heinig B, Schönlebe J et al. Debulking surgery for elephantiasis nostras with large ectatic podoplanin-negative lymphatic vessels in patients with lipo-lymphedema. Eplasty 2014; 14: e11. eCollection 2014. PMID:24741382 Free PMC article

[4] Pereira De Godoy JM, De Moura Álvares R, Simon Torati JL et al. Clinical aspects of advanced stage lipo-lymphedema: case report. G Ital Dermatol Venereol 2010; 145 (4): 547549. PMID:20823797

[5] Lohrmann C, Foeldi E, Langer M. MR imaging of the lymphatic system in patients with lipedema and lipo-lymphedema. Microvasc Res 2009; 77 (3): 335-339. doi:10.1016/j. mvr.2009.01.005. Epub 2009 Jan 27. PMID:19323976

[6] Pfister C, Dawczynski H, Schingale FJ. Selenium Deficiency in Lymphedema and Lipedema A Retrospective Cross-Sectional Study from a Specialized Clinic. Nutrients 2020; 12 (5): E1211. doi:10.3390/nu12051211. PMID:32344864

\section{Publikationshinweis}

Leserbriefe stellen nicht unbedingt die Meinung von Herausgebern oder Verlag dar. Herausgeber und Verlag behalten sich vor, Leserbriefe nicht, gekürzt oder in Auszügen zu veröffentlichen.

\section{Bibliografie}

Phlebologie 2021; 50: 3-4

Online-Publikation: 1.12.2020

DOI 10.1055/a-1217-0985

ISSN 0939-978X

(c) 2020. Thieme. All rights reserved.

Georg Thieme Verlag KG, Rüdigerstraße 14, 70469 Stuttgart, Germany 